\title{
立体视觉方法新进展 ${ }^{*}$
}

\author{
许传祥 石青云 程民德
}

(北京大学信息科学中心, 北京 100871)

\section{关键词 立体视觉 边线 轮廟线 吴方法}

精确地获取景物的三维信息是计算机视觉的重要任务之一. 双目立体视觉 (以下简称立 体视觉) 是从两个视点得到的图象来恢复出景物三维描述的技术, 可以用以下 3 个步骤来刻划立 体视觉方法:

(1) 确定匹配特征,并从图象中抽取这些特征;

(2)特征匹配, 即寻找左、右两幅图象中由景物表面上同一特征投影形成的特征对;

(3)利用对应的特征和成像几何参数重建三维景物.

经典的立体视觉方法大都是用边缘点或直线段作为匹配特征. 这些特征在图象中相对来 说比较容易检测, 但也有一些不可避免的缺点: (1) 在大多数情形下, 图象中有很多的边缘点 或直线段, 建立匹配非常困难; (2)有的时候却根本无边缘点和直线段可供选择, 例如表面光 滑的球体; (3) 这种方法得到的是景物表面稀疏的特征点, 要通过内插来重建三维景物是非常 花费时间的,而视觉系统往往要求实时处理, 因此人们开始研究整体立体视觉方法.

本文给出了一种基于吴方法的整体立体视觉方法. 吴方法 ${ }^{[1,2]}$ 是由著名数学家吴文俊先 生创立的, 它分为吴消元法和吴推理法两部分, 分别为多项式方程组的求解和空间几合关系 的机械化推理建立了完整的理论, 并提供了有效的算法. 我们把吴方法应用到立体视觉领 域, 给出了一种用二次曲线作为基元的立体视觉方法. 由于二次曲线包含了物体的整体信 息, 因此可以得到整体视觉方法. 事实上, 我们利用吴方法得到了边线型对应约束条件、轮廓 线型对应约束条件以及曲线型约束条件, 由此给出了空间二次曲面体解析形式的重建公式.

\section{1 摄像机几何模型及坐标系统描述}

立体视觉中摄像机的几何关系和坐标系统如图 1 所示.

两个摄像机的位置决定了坐标系 $o_{L}-u_{L} v_{L} w_{L}$ 和 $o_{R}-u_{R} v_{R} w_{R}$, 光心 $o_{L}$ (或 $o_{R}$ ) 是坐标系 $o_{L}-u_{L} v_{L} w_{L}$ (或 $o_{R}-u_{R} v_{R} w_{R}$ ) 的原点, $w_{L}$ (或 $w_{R}$ ) 轴是摄像机的光轴, 象平面与坐标平面 $u_{L} v_{L}$ (或 $u_{R} v_{R}$ ) 平行, 且离原点的距离为 $f_{L}$ (或 $f_{R}$ ); 为了研究方便, 我们再引人两个坐标系 $o_{L}-x_{L} y z$ 和 $o_{R}-x_{R} y z$, 光心 $o_{L}$ (或 $o_{R}$ ) 是它们的坐标原点, $x_{L}$ (或 $x_{R}$ ) 轴与基线 $o_{L} o_{R}$ 重合. 坐标系 $o_{L}-x_{L} y z$ 和 $o_{R}-x_{R} y z$ 之间的 坐标转换公式是 $x_{L}=x_{R}+D B$, 坐标系 $o_{L}-x_{L} y z$ 和 $o_{L}-u_{L} v_{L} w_{L}$ (或 $o_{R}-x_{R} y z$ 和 $o_{R}-u_{R} v_{R} w_{R}$ ) 之间的 坐标转换公式是

1994-10-05 收稿, 1995-04-25 收修改稿

*国家自然科学基金及国家“登”计划和国家教委博士点资助项目 


$$
\left(\begin{array}{l}
x_{L} \\
y \\
z
\end{array}\right)=R_{L}\left(\begin{array}{l}
u_{L} \\
v_{L} \\
w_{L}
\end{array}\right)\left(\text { 或 }\left(\begin{array}{l}
x_{R} \\
y \\
z
\end{array}\right)=R_{R}\left(\begin{array}{l}
u_{R} \\
v_{R} \\
w_{R}
\end{array}\right)\right) \text {, }
$$

其中 $R_{L}\left(\right.$ 或 $\left.R_{R}\right)$ 是旋转矩阵.

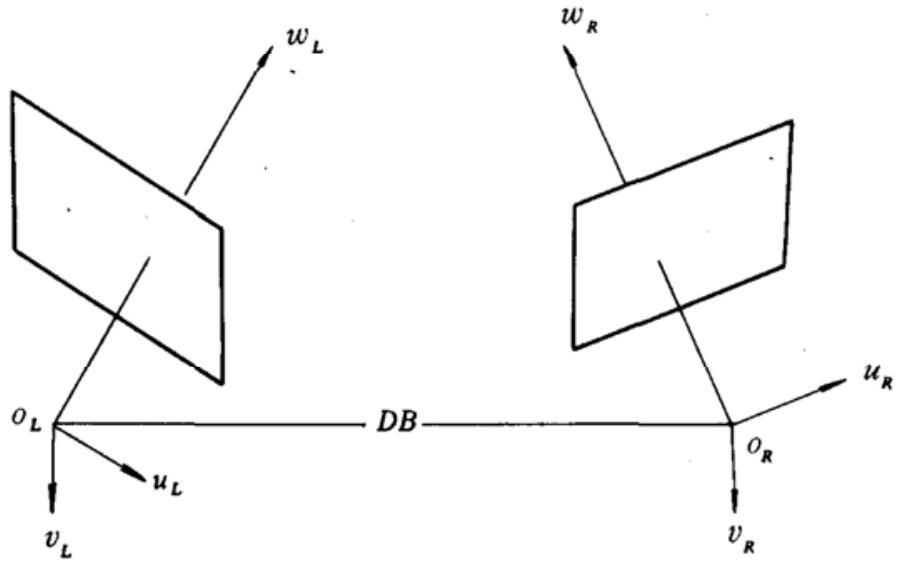

图 1 立体视觉系统摄像机几何模型

\section{2 基本概念}

定义 1 锥面 $H$ 称为曲线 $L$ 的投影锥, 如果 $H$ 是以 $L$ 为母线, 以视点为顶点的雉面. $H$ 和象平面的交线称为曲线 $L$ 的投影曲线. 特别地, 如果 $L$ 在象平面上, 则称 $H$ 为 $L$ 所形成的 视锥.

定义 2 锥面 $H$ 称为曲面 $S$ 的切雉, 如果 $H$ 是以视点为顶点的雉面, 且 $H$ 上任一过视 点的直线和曲面 $S$ 相切. $H$ 和象平面的交线称为曲面 $S$ 的轮廓线.

定义 3(边线型对应) 我们称左、右象平面上的二次曲线对 $\left(L_{L}, L_{R}\right)$ 存在边线型对应 关系, 如果存在二次曲线 $L$, 它对于左、右视点的投影曲线分别是 $L_{L}$ 和 $L_{R}$.

定义 4(轮廓线型对应) 我们称左、右像平面上的二次曲 线对 $\left(L_{L}, L_{R}\right)$ 存在轮廊线型 对应关系, 如果存在二次曲面 $S$, 它对于左、右视点的轮遊线分别是 $L_{L}$ 和 $L_{R}$.

在本文中, 我们假设线画图已从左、右图像中提取出来并且对于左 (或右)象平面上的二次 曲线 $L_{L}$ (或 $L_{R}$ ), 已经求得它们的视雉 $H_{L}$ (或 $H_{R}$ ) 在坐标系 $o_{L}-x_{L} y z$ (或 $o_{R}-x_{R} y z$ )下的方程. 我 们在此基础上给出约束条件和景物的三维重建方法.

\section{3 约束条件}

定理 1(边线型对应约束) 设 $H_{L}, H_{R}$ 分别是左、右像平面上二次曲线 $L_{L}$ 和 $L_{R}$ 的视 雉, 其方程分别是:

$$
\begin{aligned}
& H_{L}: A_{1} x_{L}^{2}+A_{2} y^{2}+A_{3} z^{2}+2 A_{4} x_{L} y+2 A_{5} x_{L} z+2 A_{6} y z=0, \\
& H_{R}: B_{1} x_{R}^{2}+B_{2} y^{2}+B_{3} z^{2}+2 B_{4} x_{R} y+2 B_{5} x_{R} z+2 B_{6} y z=0 .
\end{aligned}
$$

则 $\left(L_{L}, L_{R}\right)$ 存在边线型对应关系当且仅当以下两条件之一成立: 
(1) $A_{1} B_{1} \neq 0$ 而且 $\frac{C_{1}-C_{3}^{2}}{D_{1}-D_{3}^{2}}=\frac{C_{2}-C_{4}^{2}}{D_{2}-D_{4}^{2}}=\frac{C_{5}-C_{3} C_{4}}{D_{5}-D_{3} D_{4}}=m>0$,

其中 $C_{i}=\frac{A_{i+1}}{A_{1}} \quad D_{i}=\frac{B_{i+1}}{B_{1}} \quad i=1,2, \cdots 5$. 若此条件成立, 我们可以定义:

$$
p_{0}=1 \pm \sqrt{m}, \quad p_{1}=C_{3} \pm D_{3} \sqrt{m}, \quad p_{2}=C_{4} \pm D_{4} \sqrt{m},
$$

(2) $A_{1} B_{1}=0$, 而且下面的方程组有实数解.

$$
\frac{A_{2}-2 A_{4} p_{1}}{B_{2}}=\frac{A_{3}-2 A_{5} p_{2}}{B_{3}}=\frac{A_{6}-A_{4} p_{2}-A_{5} p_{1}}{B_{6}}=\frac{A_{4}\left(1-p_{0}\right)}{B_{4}}=\frac{A_{5}\left(1-p_{0}\right)}{B_{5}} .
$$

如果 $\left(L_{L}, L_{R}\right)$ 存在边线型对应关系, 则它所确定的二次曲线 $L$ 由下式给出:

$$
\left\{\begin{array}{l}
B_{1} x_{R}^{2}+B_{2} y^{2}+B_{3} z^{2}+2 B_{4} x_{R} y+2 B_{5} x_{R} z+2 B_{6} y z=0, \\
p_{0} x_{R}+p_{1} y+p_{2} z+D B=0 .
\end{array}\right.
$$

注 1 有时边线型对应是虚的, 即 $L$ 落在区域 $w_{L}<f$ 或 $w_{R}<f$ 之内, 我们可以通过特殊 点测试法排除这种情况.

注 2 一般说来, 可从边线型对应 $\left(L_{L}, L_{R}\right)$ 恢复出两条二次曲线, 我们可通过连续视差 假设或三目立体视觉确定其唯一性.

证 $\left(L_{L}, L_{R}\right)$ 存在边线型对应关系的充要条件是存在平面 $P$, 使得

$\left\{\begin{array}{l}H_{L}=0 \\ P=0\end{array} \Rightarrow H_{R}=0\right.$ 和 $\quad\left\{\begin{array}{l}H_{R}=0 \\ P=0\end{array} \Rightarrow H_{L}=0\right.$ 同时成立.

利用吴推理原理即可得到边线型匹配约束条件, 它是一关于 $p_{1}, p_{2}, p_{3}$ 的二次联立方程 组,通过吴消元法化简即得定理结论.

下面的定理对任何坐标系都成立, 因此我们用 $o-x y z$ 代指我们用到的任何坐标系.

定理 2(曲线型约束) 设二次曲线 $L$ 的方程为

$$
L:\left\{\begin{array}{l}
x^{2}+A_{1} y^{2}+A_{2} z^{2}+2 A_{3} x y+2 A_{4} x z+2 A_{5} y z=0, \\
q_{1} x+q_{2} y+q_{3} z+D B=0 .
\end{array}\right.
$$

而且 $\left(A_{1} q_{1}^{2}+q_{2}-2 A_{3} q_{1}\right) q_{1} \neq 0$, 则曲面 $S$ :

$$
a_{0} x^{2}+a_{1} y^{2}+a_{2} z^{2}+2 a_{3} x y+2 a_{4} x z+2 a_{5} y z+2 D B a_{6} x+2 D B a_{7} y+2 D B a_{8} z+D B^{2}=0 \text {, }
$$

经过 $L$, 当且仅当以下 5 个公式同时成立.

$$
\begin{aligned}
a_{2} & =C_{1}\left(a_{1}-2 a_{3} p_{1}+a_{0} p_{1}^{2}\right)+2 a_{4} p_{2}-a_{0} p_{2}^{2} ; \\
a_{5} & =C_{2}\left(a_{1}-2 a_{3} p_{1}+a_{0} p_{1}^{2}\right)+a_{4} p_{1}+a_{3} p_{2}-a_{0} p_{1} p_{2} ; \\
a_{7} & =C_{3}\left(a_{1}-2 a_{3} p_{1}+a_{0} p_{1}^{2}\right)+a_{6} p_{1}+a_{3} p_{3}-a_{0} p_{1} p_{3} ; \\
a_{8} & =C_{4}\left(a_{1}-2 a_{3} p_{1}+a_{0} p_{1}^{2}\right)+a_{6} p_{2}+a_{4} p_{3}-a_{0} p_{2} p_{3} ; \\
a_{0} p_{3}^{2} & =C_{5}\left(a_{1}-2 a_{3} p_{1}+a_{0} p_{1}^{2}\right)+2 a_{6} p_{3}-1 .
\end{aligned}
$$

证通过简单计算可以求出 $L$ 的不可约升列 $I R R$, 由吴方法中的母元法理论知, 二次曲 面 $S$ 经过 $L$ 的充要条件是 $S$ 对 $I R R$ 求余余式为零, 直接计算即得定理结论.

定理 3(轮廊线型约束) 条件同定理 1 , 如果 $A_{1} B_{1} \neq 0$ 则 $\left(L_{L}, L_{R}\right)$ 存在轮廓线型对应关 系的当且仅当 


$$
\frac{C_{1}-C_{3}^{2}}{D_{1}-D_{3}^{2}}=\frac{C_{2}-C_{4}^{2}}{D_{2}-D_{4}^{2}}=\frac{C_{5}-C_{3} C_{4}}{D_{5}-D_{3} D_{4}}=m \text {, 其中 } C_{i}=\frac{A_{i+1}}{A_{1}}, D_{i}=\frac{B_{i+1}}{B_{1}}, i=1,2, \cdots 5 .
$$

如果 $\left(L_{L}, L_{R}\right)$ 存在轮廓线型对应关系, 则它所确定的二次曲面由下式给出:

$$
a_{0} x_{R}^{2}+a_{1} y^{2}+a_{2} z^{2}+2 a_{3} x_{R} y+2 a_{4} x_{R} z+2 a_{5} y z+2 D B a_{6} x_{R}+2 D B a_{7} y+2 D B a_{8} z+D B^{2}=0 \text {, }
$$

其中 $\quad a_{0}=2 b_{6}+m+1 ; \quad a_{1}=\left(D_{3}^{2}-D_{1}\right) b_{6}^{2}+2 C_{3} D_{3} b_{6}+m D_{1}+C_{3}^{2}$;

$$
\begin{aligned}
& a_{2}=\left(D_{4}^{2}-D_{2}\right) b_{6}^{2}+2 C_{4} D_{4} b_{6}+m D_{2}+C_{4}^{2} ; \quad a_{3}=\left(C_{3}+D_{3}\right) b_{6}+C_{3}+m D_{3} ; \\
& a_{4}=\left(C_{4}+D_{4}\right) b_{6}+C_{4}+m D_{4} ; \\
& a_{5}=\left(D_{3} D_{4}-D_{5}\right) b_{6}^{2}+\left(C_{3} D_{4}+C_{4} D_{3}\right) b_{6}+m D_{5}+C_{3} C_{4} ; \\
& a_{6}=b_{6}+1 ; \quad a_{7}=D_{3} b_{6}+C_{3} ; \quad a_{8}=D_{4} b_{6}+C_{4},
\end{aligned}
$$

式中 $b_{6}$ 是一满足 $m-b_{6}^{2} \neq 0$ 参数.

注 3 有时轮廓线型对应是虚的, 即 $S$ 落在区域 $w_{L}<f$ 或 $w_{R}<f$ 之内, 我们可以通过 特殊点测试法排除这种情况.

注 4 一般说来, 可从轮廊线型对应 $\left(L_{L}, L_{R}\right)$ 恢复出带一个自由参数的二次曲面族, 这 需利用其他物理假设或三目立体视觉确定其唯一性.

注 5 如果 $m>0$, 那么无法区分边线型对应与轮廓线型对应, 这时必需借助其他约束 条件, 如三目立体视觉.

证 $\left(L_{L}, L_{R}\right)$ 存在轮廊线型对应关系的充要条件是存在二次曲面 $S$, 使得 $H S_{L}=0 \Leftrightarrow H_{L}=0$ 和 $H S_{R}=0 \Leftrightarrow H_{R}=0$ 同时成立, 其中 $H S_{L}, H S_{R}$ 分别是 $S$ 对左、右视点的切 锥,利用吴推理法可求得约束条件, 通过吴消元法化简即得定理结论.

\section{4 结论}

本文给出了一种用二次曲线作为基元的立体视觉方法. 二次曲线基元的主要优点是可以 建立整体的对应关系, 并求得解析形式的重建公式. 通过利用吴方法这一空间几何关系推理 和方程求解的强有力工具, 得到了一种非常优美且又简单的建立对应和实现景物三维重建的 方法. 理论分析和实验结果都标明此方法具有算法简单、速度快等特点.

\section{参考文献}

1 Kapur D, Mundy J L. Wu's method and its application to perspective viewing. Artificial Intelligence, 1988, 37 : 15 36

2 Koh K, Deguchi K. Recognition of polyhedra by a mechanical theorem proving method. First Korean-Japanese Joint Conferenœ on Commuter Vision, 1991. $144 \sim 151$ 